\title{
Massive Data Oriented Replication Algorithms for Consistency Maintenance in Data Grids
}

\author{
Changqin Huang, Fuyin $\mathrm{Xu}$, and Xiaoyong $\mathrm{Hu}$ \\ College of Educational Information Technology, South China Normal University, \\ Guangzhou, 510631, P.R. China \\ cqhuang@zju.edu.cn, xufy@scnu.edu.cn, huxiaoy@hotmail.com
}

\begin{abstract}
Based on the Grid Community (GC) for data usage, differentiated replication algorithms are proposed, by which the replica node is selected according to key replica node $(S H N)$ and the other. First, The replicas are passed into $S H N$; second, if certain nodes in a GC often access the data resource, it or its nearby nodes become an optional replica node based on the storage and bandwidth. A consistency maintenance algorithm is presented to facilitate the coherence function in a differentiated manner: a pessimistic manner or an optimistic manner, and to update according to the context. The simulation results show that the differentiated mechanism can improve grid access performance.
\end{abstract}

\section{Introduction}

In Data Grids, the management of massive data is one of the major scientific challenges. Data replication is an important enabling technology as grid services. Replication improves availability and performance, and increase throughput. However, Data replication also brings a few hard-solved issues, such as consistency maintenance. To do well the trade-offs among performance and consistency, we present a differentiated replication approach including Optimistic replication [1].

\section{The Differentiated Replication Algorithms}

Because any large amount of data is produced by a single resource, we think that there is only a steady original data, named home data that resides in a certain Home Node $(H N)$. Replicas exchange updates in a peer-to-peer fashion. The replicas have loosely synchronized clocks. Generally, the whole grid system consists of few Grid Communities (GC), which are perhaps a WAN or a group of nearby LANs. The GC is an important network entity, in which there exists a key node for performance as to replication, and its network connecting with the $H N$ is better. The key node should store a Secondary Home Replica $(S H R)$, and the node is called $S H N$, Except the $S H N$, The other nodes where the common replicas exist are not special to impact on each other's performance. The replica distribution is structured as Figure 1.

Due to the topology fact, our algorithms are to put replicas in a differentiated manner. First, pass replicas into key nodes, $S H N$. Second, if a certain node in a GC often accesses home replica or replica and its storage (or its nearby node) permission, a replica is stored into the node (or the nearby node). We assume the bandwidth of each 
node is available in advance, and each node knows its nearby nodes. The $A N$ denotes the number of access to home replica or replica. $T$ indicates a threshold value of $A N$. If the $A N$ exceeds $T$, the system should put a replica locally to improve performance. At present, the $S H N$ and $T$ are given in advance. Re.NodeID denotes the node ID, which produces a request for replica. The algorithm is described in Figure 2.

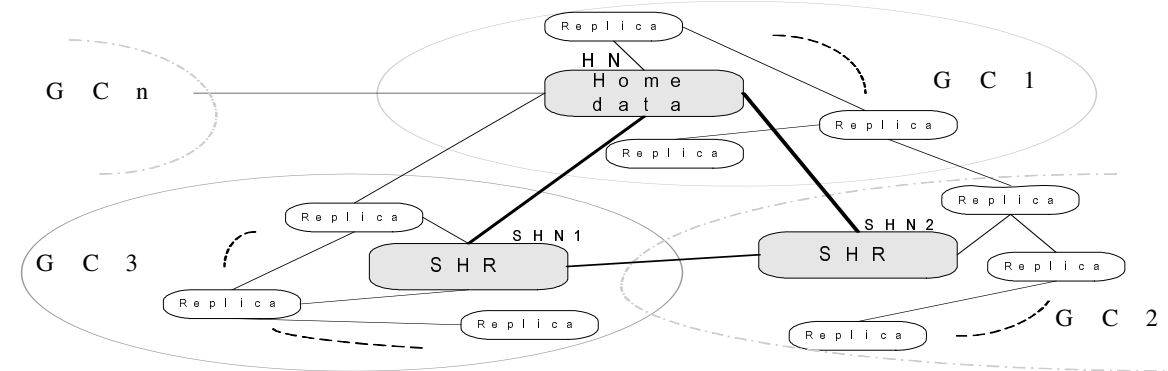

Fig. 1. The topology of replica distribution under the differentiated replication algorithms

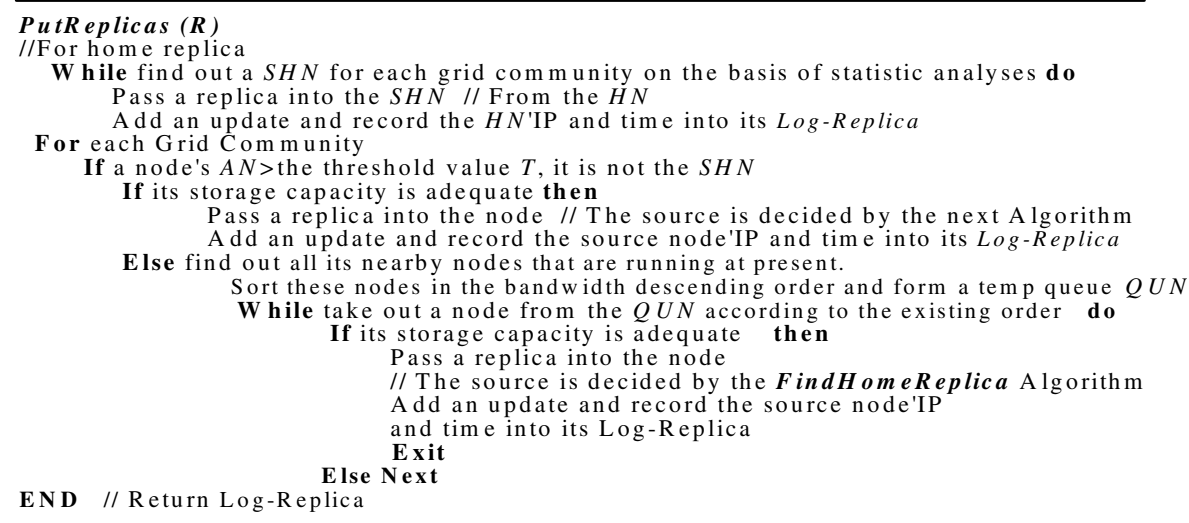

Fig. 2. The differentiated replication algorithm

\section{FindHomeReplicas $($ Re $)$}

//For each request for replica source

If Re.NodeID is the $S H N$ in the associated grid community then

Return $S H N$ (The home data is stored here)

Else find out all nodes near Re.NodeID if these nodes are running at present.

Sort these nodes in the bandwidth descending order and form a temp queue $Q U N$

While take out a node $T a N$ from the $Q U N$ according to the existing order do
If a certain Log-Replica exists in the TaN then
Return $T a N$
Exit
Else Next

End // Return TaN

Fig. 3. The find home replicas algorithm of the differentiated replication 
The system introduces a XML log file for replica, called Log-Replica, which is a key basis of replica updating and update tracing. Its existence indicates the replica existence in a hosting node. Because the Log-Replica is XML format, it is common to be applied in many aspects. And X-Diff [2] is applied to produce an analysis data, and to give the information of newly updates.

\section{The Consistency Maintenance Algorithm}

If the diff is not available during updating, we directly transfer the whole copy of the home. The system also facilities the function in a differentiated manner: a pessimistic manner and an optimistic manner. As to these SHNs, A notification mechanisms enforced. When a home replica is copied into a $S H N$, the $S H N$ completes the update subscription. The $H N$ performs an update-based publication. The action is based on message mechanisms. As for the other grid nodes of replica, an optimistic update is invoked: the system visit the metadata to get the "time" of the home and that replica, then compare these two values to decide to take relevant actions.

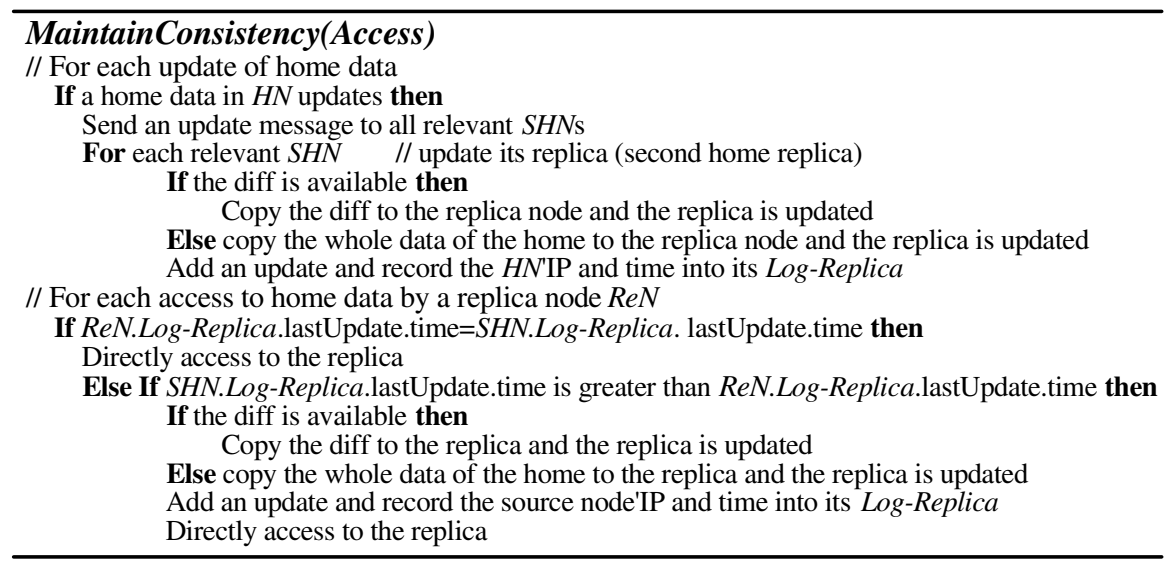

Fig. 4. The consistency maintenance algorithm

\section{Simulations}

We adopt OptorSim [3] to simulate these applications. In our simulation, parameters are set as follows: The size of each data object is $0.5 \mathrm{~GB}$ and each update is $50 \mathrm{MB}$. The time required to send a message from one replica to another is assumed to be negligible compared to the time between anti-entropy sessions. Experiments are described as follows: Case 1: The grid nodes of replica are identical to make replica and consistency maintenance; Case 2: Making replica and consistency maintenance applies our algorithms. 


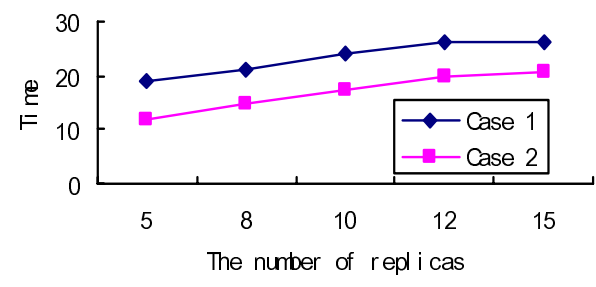

(a)

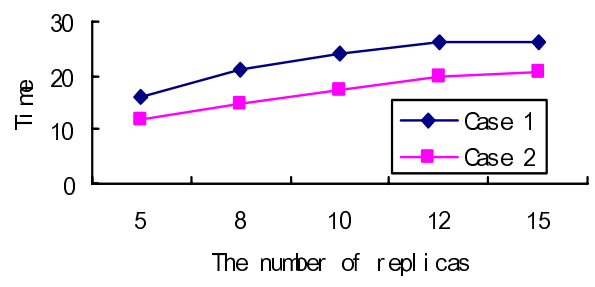

(b)

Fig. 5. (a) The time that an update has been received by all replicas for varying numbers of replicas. (b) The latency required from the time when an update is issued to the time when acknowledgments are received by each replica.

As shown in these figures, whether the first concerning time or the second, different manners for replication and updates have different performance scenarios. The Case 2 brings a better show than the Case 1, i.e. it improves data access performance that making replica and consistency maintenance applies our differentiated algorithms.

\section{Conclusions}

We present a suit of replication and consistency maintenance algorithms to meet the data grid requirements. A differentiated replication algorithm is proposed, by which the replica node is selected according two types: key replica node $(S H N)$ and the other replica nodes. Firstly, The replicas are passed into $S H N$; Secondly, if a certain node in a GC often accesses the data resource, it or its nearby nodes become an optional replica node based on bandwidth etc. Lastly, we apply a consistency maintenance algorithm to facilitate coherence function in a two manner: a pessimistic manner, an optimistic manner. The simulation results suggest that replication algorithms are fit for consistency maintenance, and can improve grid performance of data access.

Acknowledgement. The authors wish to thank the Scientific Research Fund of Hunan Provincial Education Department(Grant No. 04A037), by which the research project has been supported.

\section{References}

1. T. W. Page, Jr., R. G. Guy, J. S. Heidemann, et al: Perspectives on optimistically replicated, peer-to-peer filing. Software --Practice and Experience, vol. 28(2): 155-180, 1998.

2. Y. Wang, D. J. DeWitt, J.Y. Cai: X-Diff: An Effective Change Detection Algorithm for XML Documents, Proc. of the 19th International Conference on Data Engineering (ICDE'03), 519-530, 2003.

3. W. H. Bell, D. G. Cameron, L. Capozza, et al: Optorsim: a Grid Simulator for Studying Dynamic Data Replication Strategies. International Journal of High Performance Computing Applications, Vol. 17(4): 403-416, 2003. 